\title{
The Role of Location-Context Binding in Nonspatial Visual Working Memory
}

\author{
Ying Cai,, ${ }^{1,4}$ Jacqueline M. Fulvio, ${ }^{2}$ - Qing Yu, ${ }^{2}$ Andrew D. Sheldon, ${ }^{3,4}$ and Bradley R. Postle $e^{2,4}$
}

https://doi.org/10.1523/ENEURO.0430-20.2020

${ }^{1}$ Department of Psychology and Behavioral Science, Zhejiang University, Hangzhou 310007, China, ${ }^{2}$ Department of Psychology, University of Wisconsin-Madison, Madison, WI 53706, ${ }^{3}$ Medical Scientist Training Program and

Neuroscience Training Program, University of Wisconsin-Madison, Madison WI 53706, and ${ }^{4}$ Department of Psychiatry, University of Wisconsin-Madison, Madison, WI 53706

\begin{abstract}
Successful retrieval of an item from visual working memory (VWM) often requires an associated representation of the trial-unique context in which that item was presented. In experiment 1, fMRI of 16 male and female humans replicated a previous dissociation of the effects of manipulating memory load in comparison to the effects of manipulating context binding, by comparing VWM for one oriented line versus for three lines individuated by their location versus for three "heterogeneous" items drawn from different categories (orientation, color, and luminance): delay-period fMRI signal in frontal cortex and intraparietal sulcus (IPS) was sensitive to stimulus homogeneity rather than to memory load per se. Additionally, inspection of behavioral performance revealed a broad range of individual differences in the probability of responses to nontargets (also known as "swap errors"), and a post hoc comparison of high swap-error versus low swap-error groups generated several intriguing results: at recall, high swap-error subjects were seen to represent both the orientation and the location of the probed item less strongly, and with less differentiation from nonprobed items, and delay-period signal in IPS predicted behavioral and neural correlates of context binding at recall. In experiment 2, which was a preregistered replication, the 27 male and female humans were grouped into low and high swap-error groups by median split, and the results were broadly consistent with experiment 1 . These results present a neural correlate of swap errors, and suggest that delay-period activity of the IPS may be more important for the operation of context binding than for representation per se of stimulus identity.
\end{abstract}

Key words: fMRI

\section{Significance Statement}

Although we often think of the contents of visual working memory (VWM) as representations of the items that need to be remembered, each item's trial-unique context is also critical for successful performance. For example, if one observes a red, then a black, then a blue car passing through an intersection, vivid memory for the colors, alone, would not allow one to execute the instruction "follow the first of the three cars that just drove by." Although manipulating load is commonly assumed to isolate storage functions, requiring memory for multiple items drawn from the same category also increases demands on the context binding needed to individuate these items. This experiment tracked the influence of context binding on VWM stimulus processing.

Received October 2, 2020; accepted November 17, 2020; First published November 30, 2020.

The authors declare no competing financial interests.
Author contributions: Y.C., Q.Y., and B.R.P. designed research; Y.C., J.M.F., and A.D.S. performed research; Y.C., J.M.F., Q.Y., and A.D.S. analyzed data; Y.C., J.M.F., and B.R.P. wrote the paper. 


\section{Introduction}

Individual differences in human visual working memory (VWM) capacity result from several factors, including the strategic deployment of attention (Fukuda et al., 2015), retrieval-related processes (Unsworth et al., 2014) and resistance to interference (Vogel et al., 2005). In fMRI studies, delay-period activity in the intraparietal sulcus (IPS) scales with the number of items in the memory set before asymptoting at an individual's VWM capacity (Todd and Marois, 2004, 2005), and has often been assumed to reflect stimulus representation in VWM (Bettencourt and Xu, 2016; Xu, 2017). Here, we test the possibility that this activity may also reflect context binding: the association of each item in the memory set with its unique episodic context. In tests that require VWM for an array of colored squares, for example, the subject must remember not only each of the colors, but also the location at which each color was presented. Intact memory for all the colors but an impaired representation of which had been presented where can lead to "swap errors" (Schneegans and Bays, 2017). Indeed, some theoretical accounts hold that context binding is the factor that determines whether a stimulus can meaningfully be said to be "in" VWM (Oberauer and Lin, 2017).

In one previous study (Gosseries et al., 2018), we sought to unconfound the storage demands of a highload condition from its context-binding demands by varying stimulus category homogeneity within the memory set: subjects were asked to remember either the direction of motion in one random-dot kinematogram (RDK; $1 \mathrm{M}$ trials), the directions in three RDKs (3 M), or the direction in one RDK plus the colors of two color patches (1M2C). Although delay-period activity of the IPS was elevated during $3 \mathrm{M}$ relative to $1 \mathrm{M}$ trials, it was comparable for $1 \mathrm{M} 2 \mathrm{C}$ and $1 \mathrm{M}$ trials, indicating sensitivity to a factor other than memory load per se. In this experiment, the critical context was ordinal position, because stimuli on three-item trials were presented serially and all in the same location, with the item to be recalled prompted by a digit indicating "first," "second," or "third."

In the present experiment, we tested context binding in VWM more directly. First, we changed the behavioral task such that stimulus location was the critical contextual dimension. Because the neural correlates of spatial processing are better understood than those of ordinal processing, this allowed a more direct assessment of variation in the representation of stimulus context. Second, we measured individual differences in swap error rate, a behavioral index of context binding, to relate variability in context binding efficacy with neural signals.

This work was supported by the Nation Institutes of Health Grant MH064498 (to B.R.P.)

Correspondence should be addressed to Bradley R. Postle at postle@ wisc.edu.

https://doi.org/10.1523/ENEURO.0430-20.2020

Copyright @ 2020 Cai et al.

This is an open-access article distributed under the terms of the Creative Commons Attribution 4.0 International license, which permits unrestricted use, distribution and reproduction in any medium provided that the original work is properly attributed.
In a preliminary fMRI study enrolling 16 healthy young adults (experiment 1), we replicated the earlier finding that aggregated delay-period activity in IPS is more sensitive to category homogeneity in the stimulus set than to the number of items per se, reinforcing the association of this region with functions other than stimulus representation. Additionally, post hoc categorization of subject by swap error rate revealed several intriguing differences in the neural representation of stimulus content (orientation) and context (location). These gave rise to a set of preregistered hypotheses that were tested in experiment 2 . We believed that the results from the preregistered experiment (presented here as experiment 2) would provide novel insight into the interpretation of delay-period signals, and the computations underlying context-binding in VWM.

\section{Materials and Methods}

\section{Preregistered hypotheses}

In this and all subsequent sections of this manuscript, we will make the distinction between "experiment 1," the preliminary dataset and results from which our predictions arose, and "experiment 2," for which we preregistered the a priori set of hypotheses listed in this section, and the methods that we planned to use to generate and analyze de novo data to test these hypotheses. Importantly, whereas two of the hypothesis generating results from experiment 1 can be characterized as "post hoc dichotomization of participants based on a median split on scores of some variable of interest" (Yarkoni and Braver, 2010), which can be inferentially problematic, an a priori extreme groups design can be an effective way to "increase [...] power to detect effects by reducing the variance between participant groups relative to the variance within groups, thereby inflating effect sizes and making them easier to detect" (Yarkoni and Braver, 2010). (Note that for studies with preregistered hypotheses, the convention is to state these hypotheses in the Introduction or the very beginning of Materials and Methods; as a consequence, explanation of the rationale behind specific predictions, and of the names of some of the measures in which the hypotheses are framed, appear in a subsection at the end of Materials and Methods, Implementation of a priori hypothesis tests.)

\section{Hypothesis 1 (extreme-group analysis)}

The orientation recall specificity effect will be significantly greater, at TR10, for the low swap-error group than for the high swap-error group.

\section{Hypothesis 2 (extreme-group analysis)}

The location recall specificity effect will be significantly greater, at TR10, for the low swap-error group than for the high swap-error group.

\section{Hypothesis 3 (continuous analysis conducted with Spearman correlation of rank ordering)}

Individual differences in delay-period representation of stimulus locations, as assessed with multivariate inverted encoding modeling (IEM), will correlate with individual 
differences in swap-error rate (i.e., the "probability of a nontarget response," or $p \mathrm{~N}$, parameter, to be described below, Analysis of behavioral data). (Note that the choice of a Spearman correlation reflects the fact that subjects in the low swap-error group will necessarily have $p \mathrm{~N}$ values close to 0.)

\section{Hypothesis 4 (continuous analysis conducted with} Pearson correlation)

Individual differences in delay-period representation of stimulus location, operationalized as the amplitude of the IEM reconstruction of the probed item, will correlate with individual differences in the location recall specificity effect.

Precise details about how each of these hypotheses was generated and tested, and how the $n$ needed for adequate statistical power was calculated, are presented in the final subsection below, Implementation of a priori hypothesis tests.

\section{Additional analyses and "secondary hypotheses"}

In addition to the theoretically important hypotheses listed above, we planned to carry out several additional analyses that can be considered of "secondary" importance for experiment 2, because their outcomes will have little-to-no consequence for our theoretically motivated predictions. Some of these entailed replication of analyses of BOLD signal-intensity data from experiment 1, that were, themselves, replications of previous findings. Others can be thought of as being "descriptive," although they may literally entail carrying out a significance test. In this latter category, for example, it is important to determine the TRs at which the IEM reconstruction of targeted stimulus orientation is statistically reliable, for both the 10 and the 30 conditions. Furthermore, it is of interest (but not critical for hypotheses 1-4) to know whether any reliable reconstructions for the low swap-error group differ quantitatively from those of the high swap-error group. These will be identified as secondary hypotheses.

\section{Subjects}

For experiment 1, 18 right-handed volunteers [10 females, aged 18-25 years; mean $(S D)=21.70$ (1.75)] from the University of Wisconsin-Madison community participated in a behavior-only experiment for remuneration $(\$ 10 / h)$. Two of the 18 elected not to participate in the subsequent $\mathrm{fMRI}$ study, and remaining 16 subjects [eight females, aged 18-25 years; mean $(S D)=20.50$ (1.78)] participated the $\mathrm{fMRI}$ experiment for additional remuneration $(\$ 20 / h)$. All subjects provided informed consent according to the procedures approved by the Health Sciences Institutional Review Board at the University of Wisconsin-Madison. Subjects had normal or corrected-to-normal vision, no reported history of neurologic or psychiatric disease, and, for the $\mathrm{AMRI}$ experiment, no contraindications for MRI.

For experiment 2, we conducted an extreme-group analysis in which the initial step was to recruit healthy adult volunteers within the age range of 18-25 years, from the University of Wisconsin-Madison community, for a behavioral screening session comprising 100 trials of delayed recall (also known as "delayed estimation") of orientation with a set size of three. Stimuli and procedures closely followed those from 30 trials from experiment 1 (see below, Stimuli). The resultant recall data were fit with the three-factor mixture model described below (Behavioral tasks), and subjects with swap error rates of $<5 \%$ were selected for the $\mathrm{fMRI}$ sessions as part of the low swap-error group, and subjects with swap error rates $>12 \%$ were selected as part of the high swap-error group. The preregistered plan called for between 11 and 18 subjects for the low swap-error group, and between 11 and 18 subjects for the high swap-error group. The $N$ s required for each group were planned as ranges, to reflect the following factors (detailed below, Implementation of a priori hypothesis tests): (1) power analysis indicated that 29 subjects were required to achieve $90 \%$ power to detect the effect predicted by hypothesis 3 , but because this was a continuous analysis, it did not constrain how many of these 29 needed to belong to either extreme group; and (2) power analysis for a secondary hypothesis indicated that 11 subjects were needed to achieve $90 \%$ power to observe a significant reconstruction of stimulus orientation at TR10, in occipital cortex, on 30 trials. Because all other a priori estimated Ns were lower than these numbers, our stopping point for screening was when we had successfully recruited and tested a minimum of 11 subjects with swap error rates of $<5 \%$, a minimum of 11 subjects with swap error rates of $>12 \%$ (selected to ensure reasonable balance between the two groups), and a total of 29 subjects. (All subjects were paid at the end of the behavioral testing session and departed from the laboratory before their data were analyzed. They were told that they might be subsequently recontacted to be invited to participate in an fMRI study. Those re-contacted for the fMRI sessions of experiment 2 were not informed of how they had been categorized with regard to $p \mathrm{~N}$, nor were they informed about the concept or definition of a swap error. It was also possible that individuals whose $p \mathrm{~N}$ excluded them from experiment 2 could be recontacted to participate in a different study being conducted by our research group.)

Note that, because it is measures of $p \mathrm{~N}$ acquired during the $\mathrm{fMRI}$ session that we used to test our hypotheses, there were two possible developments that could have required us to recruit and behaviorally screen more individuals than the number listed above. The first was if the number of subjects whose $p \mathrm{~N}$ from the fMRI scanning session satisfying the criterion for the low swap-error group dropped below 11, and the second was if any subject's fMRI data were unusable, whether because of withdrawal from the study or poor data quality.

\section{Stimuli}

In experiment 1 there were three trial types: delayed recall (also known as "delayed estimation") of an oriented bar (10), of one from a memory set of three oriented bars (30), or of one item from a memory set of 1 oriented bar, 1 color patch, and 1 luminance patch (101C1L). Orientedbar stimuli (length, $4^{\circ}$; width, $0.08^{\circ}$ ) were rendered as the 


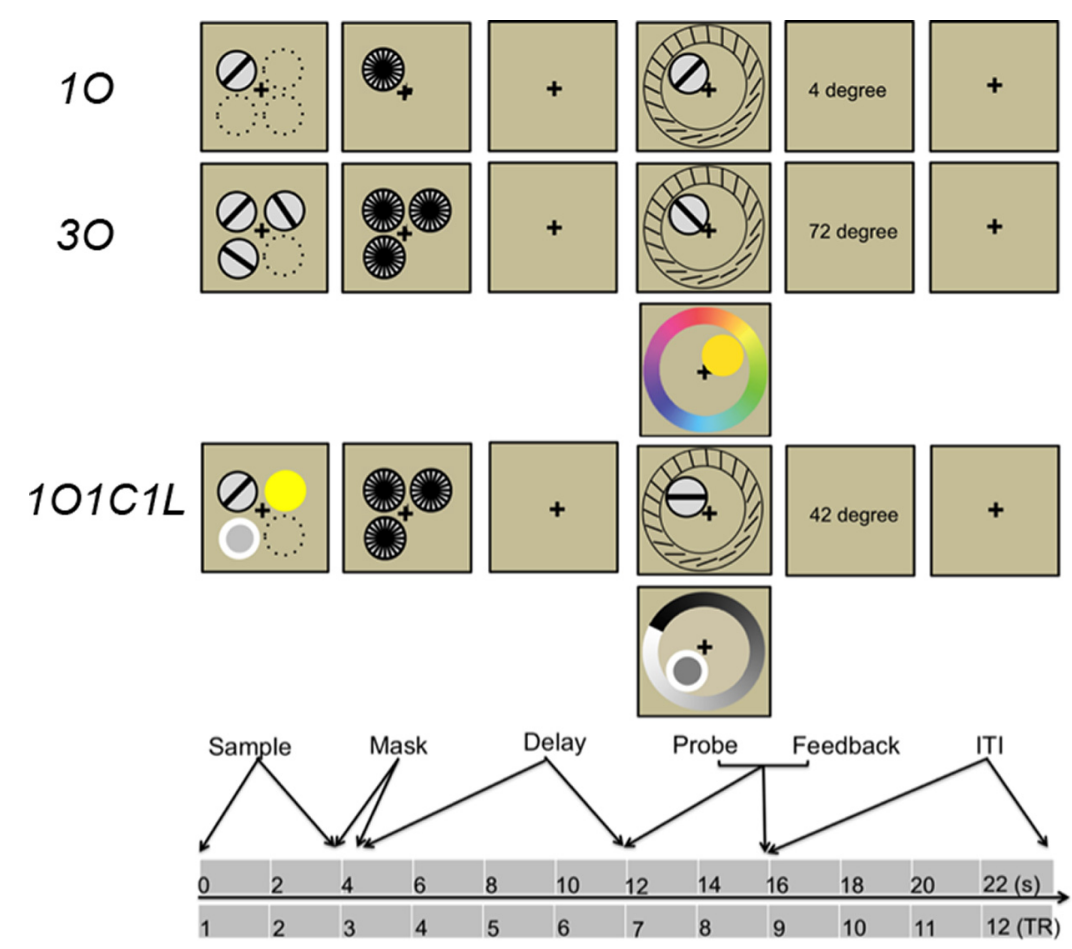

Figure 1. Schematic illustration of the three trial types from experiment $1(1 \mathrm{O}=$ one orientation; $30=$ three orientations; $101 \mathrm{C} 1 \mathrm{~L}=$ one orientation, one color, one luminance). Dotted circles indicate the other possible stimulus presentation locations, but were not presented during experiment. Experiment 2 did not include 101C1L trials.

black diameter of a white circular patch. Sample stimuli could appear in one of nine possible orientations ranging from $0^{\circ}$ to $160^{\circ}$, in $20^{\circ}$ increments, with a jitter of $\pm 1-5^{\circ}$ determined randomly on each trial. Color stimuli were presented on $4^{\circ}$-diameter circles, and drawn from a pool of 9 colors that were equidistant along a circle in $\mathrm{CIE} L^{*} a^{*} b^{*}$ color space $(L=70, a=20, b=38$, radius of 60 ; sample items were therefore equiluminant, varying mainly in hue and slightly in saturation), with a randomized jitter of $\pm 1-5^{\circ}$ on each trial. Luminance stimuli comprised a gray annulus (diameter $=2.67^{\circ}$ ) inside a white ring (RGB values ([0, 0, 0]; diameter $\left.=4^{\circ}\right)$. The annulus could take on one of nine grayscale values ranging equidistantly from light gray $[0.03,0.03,0.03]$ to darkest gray $[0.97,0.97,0.97]$, with jitter of $\pm 1-5^{\circ}$ determined randomly on each trial.

On all trials, masks were rendered as a white circular patch bisected by 18 black $0.08^{\circ} \times 4^{\circ}$ bars, all intersecting at their midpoints and each separated in orientation by $10^{\circ}$.

Recall displays comprised a circular stimulus patch, initially "empty," and a response wheel centered on fixation, with a radius to its outer edge of $9.2^{\circ}$ and a width of $2^{\circ}$. Varying continuously around the response wheel were all possible values of the category being tested. For orientation, this was rendered as 20 equally spaced black bars $\left(0.05^{\circ} \times 1.8^{\circ}\right)$, ranging in orientation from $0^{\circ}$ to $171^{\circ}$, in $9^{\circ}$ increments. For color and luminance, all 180 values of that dimension were evenly distributed along the circle. The angle of rotation of the response wheel varied unpredictably from trial-to-trial, to discourage response planning during the delay. At the onset of the recall display a cursor (a conventional "mouse" arrow) was always positioned at central fixation, and the stimulus patch was rendered with a randomly determined value rendered in the format of the sample stimuli. As soon as the subject began to move the trackball of the response box (see Behavioral tasks) the cursor moved correspondingly, and the stimulus patch took on the value corresponding to the location on the response wheel that was nearest to the cursor. Throughout the experiment, the background screen color was gray [0.5, 0.5, 0.5] (Fig. 1).

Experiment 2 included only 10 and 30 trial types (for rationale, see below, Behavioral task during $\mathrm{fMRI}$ scanning), and the stimuli differed from experiment 1 in that sample stimuli appeared in one of six possible locations, with orientations ranging from $0^{\circ}$ to $150^{\circ}$, in $30^{\circ}$ increments, with a jitter of $\pm 1-5^{\circ}$ determined randomly on each trial.

\section{Behavioral task}

In experiment 1 , each trial of the 10 task began with the $4 \mathrm{~s}$ presentation of a sample item equiprobably and unpredictably at one of four possible locations, each in one quadrant of the screen, and each with horizontal and vertical eccentricities from fixation of $5^{\circ}$. The identity of the sample, drawn equiprobably and unpredictably from a pool of nine orientations, varied independently of location. The 8-s delay period began with a mask presented at the same location as the sample. Responses were made by moving a cursor with a trackball and "clicking" on the recalled orientation with a button press. As soon as the 
trackball began to move, a bar appeared within the circular patch with an orientation, updating in real-time, that matched the orientation on the wheel that was closest to the cursor. RT was computed as the latency between response-wheel movement onset and button press. Feedback, indicating the error between the recalled orientation and the sample orientation (in degrees) was presented centrally, replacing the fixation cross, appearing immediately after the response until the end of the 4-s response window. Intertrial interval (ITI) was $2 \mathrm{~s}$ for the behavior-only experiment, and $8 \mathrm{~s}$ for the $\mathrm{fMRI}$ experiment. A black fixation cross was present at the center of the screen throughout each block of trials, and subjects were instructed to fixate it throughout the block.

30 trials followed the same procedure was as 10, with the following adjustments necessitated by the greater number of items: on each trial, each of the three sample stimuli was drawn randomly from the pool of nine, without replacement; the three were displayed simultaneously, with each of the four possible configurations of location occurring equiprobably and unpredictably; each of the three stimulus locations was backward masked; and the location of the probed item occurred equiprobably and unpredictably. $101 \mathrm{C} 1 \mathrm{~L}$ followed the same procedure was as for 30 , except that each trial featured one sample item drawn from each of the three stimulus categories, and the category of the probed item occurred equiprobably and unpredictably (Fig. 1).

In experiment 2, the task procedure was the same as that in experiment 1 , with two exceptions: only 10 and 30 trials were included, and stimuli could appear at six evenly spaced locations along the imaginary circumference of a circle $5^{\circ}$ in diameter, centered on fixation.

\section{Behavior-only experiment from experiment 1}

Testing was broken into two blocks of 10 and 30 trials and three blocks of $101 \mathrm{C} 1 \mathrm{~L}$ trials. All blocks contained 50 trials, and block order was counterbalanced across subjects by drawing the first 18 orders from a Latin square. Each block of 10 and 30 trials presented 25 of each, in a randomized sequence (thereby yielding a total of 5010 responses and 5030 responses per subject), and each block of $101 \mathrm{C} 1 \mathrm{~L}$ trials included 17 probes of two of the categories and 16 of the remaining category, randomized within block and balanced across the three blocks, thereby yielding $50101 \mathrm{C} 1 \mathrm{~L}$ orientation responses, $50101 \mathrm{C} 1 \mathrm{~L}$ color responses, and $50101 \mathrm{C} 1 \mathrm{~L}$ luminance responses. All the experimental stimuli were controlled by the Psychophysics Toolbox (http://psychtoolbox.org; Brainard, 1997) running in MATLAB (MathWorks), presented on a $60-\mathrm{Hz}$ projector with a screen width of $32.5 \mathrm{~cm}$ (iMac). The viewing distance was $62 \mathrm{~cm}$.

\section{Behavioral screening for experiment 2}

Procedures for the orientation WM task were identical to those from experiment 1 , with the exception that subjects only performed two 50 -trial blocks of the 30 task.

\section{Behavioral task during $\mathrm{fMRI}$ scanning}

For experiment 1, there were two scanning sessions, and during the first session subjects first performed four blocks of three-item trials: nine trials of 30 and nine trials $101 \mathrm{C} 1 \mathrm{~L}$ in a randomly determined order during each block; three probes of each category on $101 \mathrm{C} 1 \mathrm{~L}$ trials in a randomly determined order during each block. Next, subjects completed eight 18-trial blocks of 10 trials, with each orientation appearing twice in a randomly determined order during each block. In the second fMRI scanning session, subjects performed an additional 12 18-trial blocks of 10 trials. (The larger number of 10 trials was needed to train the IEM models with which data from all trial types would be tested.)

For experiment 2, subjects performed only 10 and 30 trials. The rationale was that the most novel effects observed in experiment 1 were the differences in IEM reconstruction of orientation between low versus high swaperror subjects. These differences were of greatest theoretical significance on 30 trials, which place the greatest demands on context binding. Furthermore, the results from experiment 1 involving comparison of patterns of BOLD signal intensity in the 10 versus $101 \mathrm{C} 1 \mathrm{~L}$ versus 30 conditions, and their relation to behavior (Fig. 2), were robust and adequately powered at $n=16$, and were, themselves, replications of a previous result (Gosseries et al., 2018). Therefore, for the experiment 2, we maximized the sensitivity of within-subject IEM reconstructions, and of between-group comparisons, by administering 10830 trials (an increase from the 36 that featured in experiment 1).

For the fMRI portion of experiment 2, each subject completed two 2-h scanning sessions, each on a separate day. The first scanning session began with six 18-trial blocks of 30 trials, followed by seven blocks of 10 trials. The second scanning session consisted of thirteen 18trial blocks of 10 trials. Each block of trials was performed during a 7.2-min scan. Thus, each scanning session entailed $1 \mathrm{~h} 33$ min $36 \mathrm{~s}$ of fMRI scanning, not including the brief pauses between scans. Over the course of all 108 30 trials, each orientation was probed at each location three times. Over the course of all 36010 trials, each orientation was presented (and probed) at each location a total of ten times at each location.

\section{Analysis of behavioral data}

For experiment 1, reaction time (RT) of the responseending button press was collected and raw response error distance estimated by the distance on the response wheel between the subjects' selection and the true target value (in degrees). Trials without responses were excluded. For 10 and $101 \mathrm{C} 1 \mathrm{~L}$ trials, response error was fit to a two-factor mixture model that estimated the proportion of responses made to the sample (i.e., the probability of a target response ( $p T)$, and the probability of guess responses $(\mathrm{pU})$, as well as the precision of target responses $(\kappa$; Bays et al., 2009). For 30 trials a third factor, the probability of a response to a nontarget $(\mathrm{pN}$; also known as "swap error"), was included in the model. Parameter estimates were obtained using maximum-likelihood estimation (expectation maximization) using MATLAB routines available at http://www.bayslab.com. Differences across trial type in descriptive measures and in model parameters were assessed with repeated measures one-way ANOVAs, and significant effects were followed up with 

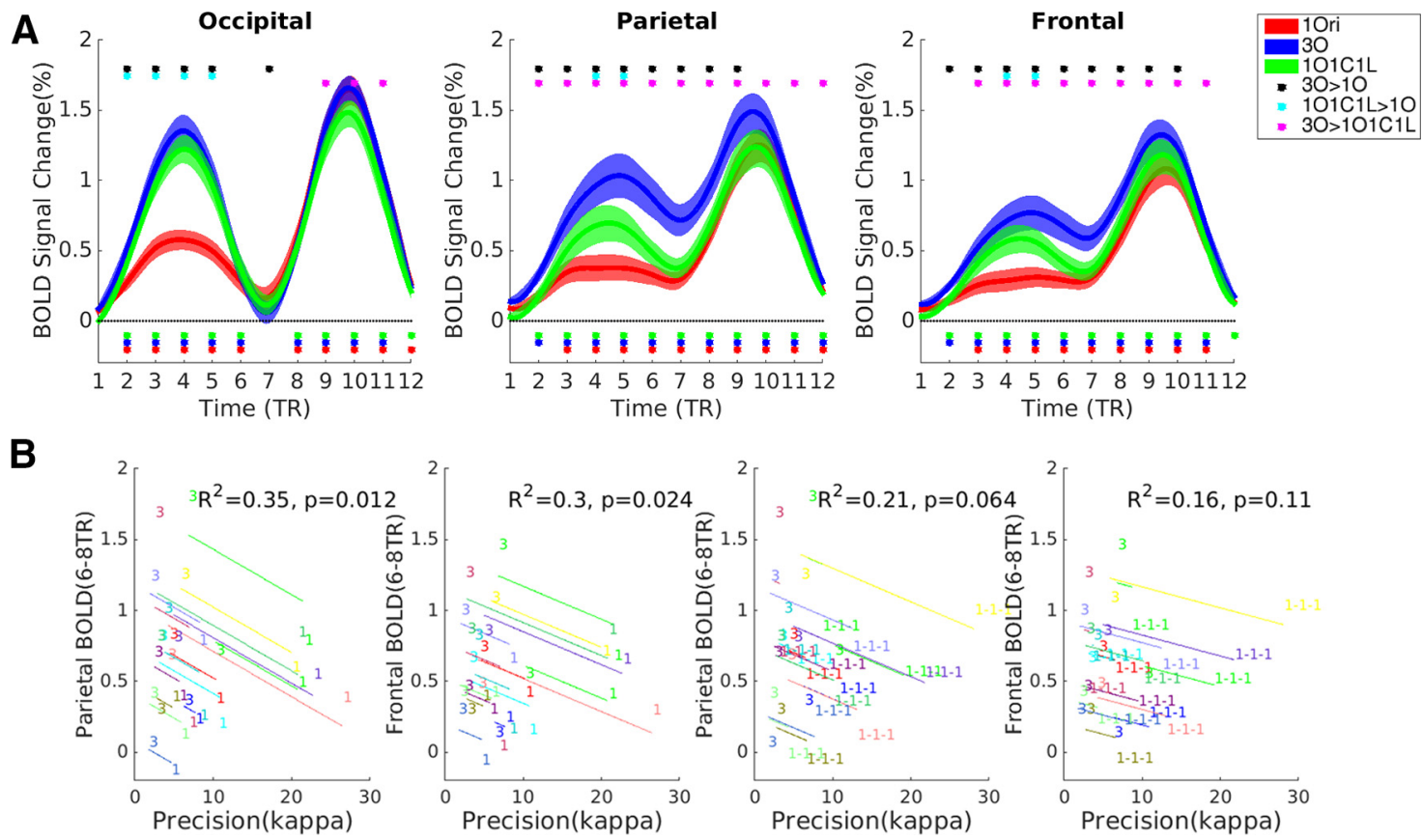

Figure 2. BOLD signal intensity results from three ROls in experiment 1 . $\boldsymbol{A}$, Trial-averaged BOLD signal. Dots below the $x$-axis indicate significance versus baseline; dots above the plots indicate significant differences between trial types. $\boldsymbol{B}$, Within-subject correlations (ANCOVAs) between delay-period BOLD signal intensity and behavioral precision of recall. In each plot, data from each subject are portrayed in a different color. The "1," "3," and "1-1-1" symbols indicate individual values in the 10, 30 , and 101C1L tasks, respectively. Thus, for example, the left-most plot illustrates that on 30 trials, delay-period BOLD signal in IPS is high and precision is low, whereas on 10 trials, delay-period BOLD signal in IPS is low and precision is high. The slope of the lines indicates the tendency at the group level, and the offsets between lines indicate where different individuals sit within this space.

paired $t$ tests. For descriptive measures, we only focused on response error, because the RT was necessarily noisy because it included the time to adjust the response dial with a trackball positioned adjacent to the thigh of the supine subject. For model estimates, we focused on $\kappa$ and $p T$, because $p U$ was highly collinear with $p T$ in 10 and $101 \mathrm{C} 1 \mathrm{~L}$ trials (i.e., for the two-factor mixture model, $p \mathrm{U}+p \mathrm{~T}=1$ ). Note that $p \mathrm{~N}$ could only be estimated in 30 trials.

The $p \mathrm{~N}$ parameter estimated from 30 trials provides a measure of the efficacy of context binding, because a swap error corresponds to a trial on which the subject has forgotten the location context of the item that they are recalling. For experiment 1, post hoc inspection of performance from the behavior-only experiment revealed a clear bimodal distribution in estimates of $p \mathrm{~N}$, with six subjects having a $p \mathrm{~N}$ at or near 0 (indicating effectively no swap errors) and the remaining 10 subjects all having a $p \mathrm{~N}$ of 0.127 or higher. Based on this pattern in the behavior-only experiment, subjects were grouped into low swap-error and high swap-error groups for the analyses of the fMRI experiment.

For the behavioral task in the fMRI component of experiment 1, RT and response errors were collected in the same way, but model fitting was only conducted for 10 trials because of the limited number of 30 and $101 \mathrm{C} 1 \mathrm{~L}$ trials. Correlations were conducted on all measures of behavioral performance on 10 trials from the two experiments, with the exception of RT, to assess the stability of subjects' performance.
For experiment 2, procedures for analyses and model fitting of data from 10 and 30 trials were the same as those from experiment 1 .

\section{fMRI methods}

Unless specified otherwise, the fMRI methods for experiment 2 followed those from experiment 1 , as described in this section.

\section{General procedure and behavioral tasks}

For experiment 1 , the fMRI experiment comprised two scanning sessions, each lasting $\sim 1.5 \mathrm{~h}$. The first of the two scanning sessions followed the behavior-only task by 6-21d, and the second scanning session followed the first by $2-28 \mathrm{~d}$. Scanning of three-item trials preceded scanning of 10 trials to minimize the likelihood that subjects would process orientation stimuli different from color and luminance stimuli on 101C1L trials. All the experimental stimuli were controlled by the Psychophysics Toolbox (http://psychtoolbox.org; Brainard, 1997) running in MATLAB (MathWorks), presented via a $60-\mathrm{Hz}$ projector (Silent Vision 6011; Avotec) backprojecting onto a screen mounted inside the bore of the scanner, and viewed through a coil-mounted mirror. The viewing distance was $68.58 \mathrm{~cm}$ and screen width was $33.02 \mathrm{~cm}$.

\section{Data acquisition}

Whole-brain images were acquired with a 3 Tesla scanner (Discovery MR750; GE Healthcare) at the Lane 
Neuroimaging Lab at the University of WisconsinMadison. For all subjects, a high-resolution T1-weighted image was acquired with a fast spoiled gradient-recalledecho sequence $\left(\mathrm{TR}=8.2 \mathrm{~ms}, \mathrm{TE}=3.2 \mathrm{~ms}\right.$, Flip angle $=12^{\circ}$, 160 axial slices, $256 \times 256$ in-plane, $1 \mathrm{~mm}$ isotropic). A T2*-weighted gradient echo pulse sequence was used to acquire data sensitive to the BOLD signal while subjects performed the VSTM task (TR $=2000 \mathrm{~ms}$, TE $=25 \mathrm{~ms}$, flip angle $=60^{\circ}$, within a $64 \times 64$ matrix, 42 sagittal slices, 3 $\mathrm{mm}$ isotropic). Each of the twenty $\mathrm{fMRI}$ scanning runs generated 213 volumes (excluding disdaqs).

\section{Preprocessing}

$\mathrm{fMRI}$ data were preprocessed using the Analysis of Functional Neuroimages (AFNI) software package (http:// afni.nimh.nih.gov; Cox, 1996). All volumes were spatially aligned to the first volume of the first run using rigid-body realignment, then aligned to the $\mathrm{T} 1$ volume. Volumes were corrected for slice-time acquisition, and linear, quadratic, and cubic trends were removed from each run to reduce the influence of scanner drift. For univariate analyses, data were spatially smoothed with a 4-mm full-width at half-maximal Gaussian, and z-scored separately within run for each voxel. For IEM and MVPA analyses (see below), data were $z$-scored separately within run for each voxel, but were not smoothed. All analyses were conducted in each subject's native space.

\section{Data analysis}

Univariate analyses and ROI creation. For experiment 1, a modified general linear model (GLM) was fit to data from all 30 and $101 \mathrm{C} 1 \mathrm{~L}$ trials and from 36 randomly selected 10 trials. It included regressors modeling the sample presentation, delay, and recall periods with boxcars of 4,8 , and $4 \mathrm{~s}$, respectively, each convolved with the canonical hemodynamic response function supplied with AFNI.

A different set of anatomically constrained, functionally defined regions of interest (ROIs) was created for each of the three categories of analysis: BOLD signal intensity, IEM of stimulus orientation, and MVPA of stimulus location. For all three, anatomic regions were generated from the standard anatomic masks for occipital, parietal, and frontal cortex from the MNI152_T1_1mm template and warping them to each subject's native space. For BOLD signal intensity analyses, an occipital sample ROI was generated for each subject by selecting the 400 voxels with the highest $t$ values for the contrast [Sample [So - $^{-}$ Sample $_{10}$ ] within anatomically defined occipital cortex, and parietal delay and frontal delay ROls were generated by selecting the 400 voxels with the highest $t$ values for the contrast [Delay ${ }_{30}-$ Delay $_{10}$ ] within each of these anatomically defined regions. Within each of these ROls, trialaveraged time series for each of the three trial types were generated and converted to mean percentage signal change from baseline (first TR of the trial), and contrasts versus baseline and between conditions conducted with $t$ tests (all $p$ values false discovery rate (FDR)-corrected across TRs, ROls, and comparisons). For IEM analyses, four sets of "sample location-specific" ROls were generated with the top 400 voxels within each of the three anatomic regions responding to the contrasts [Sample ${ }_{\text {upper left }}$ - baseline],
[Sample upper right $_{\text {- baseline], [Sample }}$ lower left - baseline], and [Sample lower right - baseline]. Finally, for MVPA analyses, "location-general" ROls were created with the top 400 voxels within each of the three anatomic regions identified with the contrast $\left[\right.$ Sample $_{\text {upper left }}+$ Sample $_{\text {upper right }}+$ Sample $_{\text {lower left }}+$ Sample lower right $_{\text {- }}$ - baseline]. (Analyses of fMRI data from 10 trials, unrelated to those described here, are presented in Cai et al. (2019)).

For experiment 2, ROI creation differed only in that it used data from all 30 trials and from 108 randomly selected 10 trials.

Task-related patterns of covariation. For experiment 1 , we used ANCOVA to evaluate evidence for correlated sensitivity across trial types (specifically, across 10 vs 30 and across 101C1L vs 30) of two of dependent variables, BOLD signal intensity and behavioral precision, seen to covary in previous studies (Emrich et al., 2013; Gosseries et al., 2018). Unlike simple correlations, ANCOVA accommodates the fact that each subject contributes a value for each level of the factor of trial type. It removes betweensubject differences and assesses evidence for "withinsubject correlation," the extent to which variation in one dependent variable can be explained by variation in a second (Bland and Altman, 1995). For analyses including delay-period $\mathrm{fMRI}$ activity, the BOLD signal was averaged across TR6 and TR7, those least likely to be contaminated by sample-related signal.

For the experiment 2, comparable ANCOVAs, conducted with data from the 30 and 10 trials, were considered secondary hypotheses.

Multivariate IEM. In experiment 1, we estimated population-level neural representations of the orientation of oriented-bar stimuli with multivariate IEM (Serences and Saproo, 2012; Sprague et al., 2018). To optimize estimation from 10 trials, four IEMs were trained within each of the three anatomic ROIs, one for each location at which a sample could appear on the screen. The four resultant location-specific reconstructions were averaged before assessment of the results of IEM training and testing.

To build our IEMs we assumed that the responses of each voxel can be characterized by activity in 9 hypothesized tuning channels, one corresponding to each of the 9 possible sample orientations. Following previous work (Ester et al., 2015; Yu and Shim, 2017), the idealized feature tuning curve of each channel was defined as a halfwave-rectified and squared sinusoid raised to the seventh power. Before feeding the preprocessed data into the IEM, a baseline from each voxel's response was removed in each run using the following equation from Brouwer and Heeger (2011):

$$
B=B-m\left(m^{T} B\right),
$$

in which $B$ represented the data matrix from each run with size $v \times c(v$ : the 400 location-specific voxels; $c$ : the nine orientations) and $m$ represented the mean response across all stimulus conditions of length $v$. Next, for trials corresponding to each of the four sample locations, we randomly divided the data into a training set (81 trials) and a test set (nine trials; these numbers were selected to 
match the total number of $101 \mathrm{C} 1 \mathrm{~L}$ and 30 trials). We computed the weight matrix $(W)$ that projects the hypothesized channel responses $\left(C_{1}\right)$ to actual measured fMRI signals in the training dataset (B1), and extracted the estimated channel responses $\left(\hat{C}_{2}\right)$ for the test dataset (B2) using this weight matrix. The relationship between the training dataset $(B 1, v \times n, n$ : the number of repeated measurements) and the channel responses $\left(C_{1}, k \times n\right)$ was characterized by:

$$
B_{1}=W C_{1},
$$

where $W$ was the weight matrix $(v \times k)$.

Next, the least-squared estimate of the weight matrix $(\hat{W})$ was calculated using linear regression:

$$
\hat{W}=B_{1} C_{1}^{T}\left(C_{1} C_{1}^{T}\right)^{-1} .
$$

The channel responses $\left(\hat{C}_{2}\right)$ for the test dataset (B2) were then estimated using the weight matrix $(\hat{W})$ :

$$
\hat{C}_{2}=\left(\hat{W}^{T} \hat{W}\right)^{-1} \hat{W}^{T} B_{2} .
$$

Having thus estimated the weight matrix mapping each voxel's response to each orientation channel from the training dataset, we inverted this matrix to estimate channel responses on each test trial. The average response output for each channel across trials was obtained by circularly shifting each response to a common center of $0^{\circ}$. To generate smooth, 180-point channel tuning functions (CTF, also referred to as "reconstructions") we repeated the encoding model analysis 180 times and shifted the centers of the orientation channels by $1^{\circ}$ on each iteration (Brouwer and Heeger, 2009). The CTFs were averaged across permutations and averaged across the four location-specific models. The same weight matrix trained in this fashion on data from 10 trials was used to reconstruct the neural representation of orientation in 10, 30, and $101 \mathrm{C} 1 \mathrm{~L}$ trials. More specifically, within each ROI, separate IEMs were trained on 10 data from each time point in the trial and then tested (i.e., reconstructions attempted) at same time point with data from 10,30, and $101 \mathrm{C} 1 \mathrm{~L}$ trials separately. For 30 trials, the location-specific IEM to be used for IEM testing was assigned according to the orientation to be tested for recall. For $101 \mathrm{C} 1 \mathrm{~L}$ trials, testing was conducted with the location-specific IEM congruent with the location occupied by the oriented bar in the sample array.

To quantify the results, the CTF in each ROI for each subject was fit with an exponentiated cosine function of the form:

$$
f(x)=\alpha\left(e^{\mathrm{k}}[\cos (\mu-x)-1]\right)+\beta .
$$

Here, $\alpha$ and $\beta$ control the vertical scaling (i.e., signal over baseline) and baseline of the function, respectively, and $\kappa$ and $\mu$ control the concentration (the inverse of dispersion) and center of the function, respectively. No biases in reconstruction centers were expected or observed, so we fixed $\mu$ at 0 . Fitting was performed by combining a GLM with a grid search procedure. We first defined a range of plausible $\kappa$ values (from 1 to 30 in 0.1 increments). For each possible value of $\kappa$, we generated a response function using the fitting equation after setting $\alpha$ to 1 and $\beta$ to 0 . Next, we generated a design matrix containing the predicted response function and a constant term (i.e., a vector of $1 \mathrm{~s}$ ) and used ordinary least-squares regression to obtain estimates of $\alpha$ and $\beta$ (defined by the regression coefficient for the response function and constant term, respectively). We then selected the combination of $\kappa, \alpha$, and $\beta$ that minimized the sum of squared errors between the observed and predicted reconstructions.

In experiment 2, the same IEM procedure was used to estimate neural representations of stimulus orientation and stimulus location, except that orientation models characterized each voxel in terms of the activity in six hypothesized orientation tuning channels (one corresponding to each possible orientation). And six separate IEMs were trained (one for each location) and these locationspecific reconstructions were averaged before assessment of the results of IEM training and testing.

\section{Implementation of a priori hypothesis tests}

Hypothesis 1 and hypothesis 2 of experiment 2 corresponded to analyses from experiment 1 conducted in the occipital ROI and focusing on TR10, one of the TRs at which CTFs in multi-item trials were reliable at the group level $(N=16)$. To carry out tests of hypotheses 1 and 2 for experiment 2, we first needed to ensure sufficient statistical power to achieve the secondary hypothesis that, for the low swap-error group, the amplitude of the reconstruction of stimulus orientation at TR10, on 30 trials, would be statistically greater than baseline. To do this, we followed our procedure from experiment 1 by assessing the significance of CTF amplitude using a bootstrapping procedure in which, for each $\mathrm{ROI}$, we randomly selected, with replacement, the number CTFs corresponding to the number of subjects in the group, and averaged them. This step was repeated 2500 times, yielding 2500 unique stimulus reconstructions. We then estimated the amplitude of each reconstruction and a $p$ value was computed as the proportion of permutations for which amplitude estimates $\leq 0$ were obtained (Ester et al., 2015, 2016). For this and all subsequent bootstrapping analyses, all the $p$ values were onetailed and FDR-corrected across ROls, TRs, and tasks. Whereas in experiment 1, Cohen's $d$ for the reconstruction of stimulus orientation at TR10 from 30 trials was 0.729 , simulations indicated that we could expect effect sizes for reconstruction of stimuli from 30 trials for experiment 2 to double relative to experiment 1 , because of the increased number of 30 trials. Therefore, to calculate the number of subjects needed in the low swap-error group to achieve $90 \%$ power for this secondary hypothesis, we used an estimate of Cohen's $d$ of $[0.729 \times 2=1.458]$. To achieve $90 \%$ power to detect this effect with $\alpha=0.025$, we needed an $N$ of 11. (Note that, because none of the primary hypotheses tested in experiment 2 required significant reconstruction of orientation in the occipital ROI at TR10 in the high swaperror group, we did not carry out power analyses for this effect.)

Additional secondary hypotheses were whether, at any TR of the task, orientation reconstructions differed between the low swap-error and high swap-error groups. 
These were assessed by first carrying out the bootstrapping procedure separately for each group, then comparing the CTF estimates from each permutation with each group by subtracting the amplitude from the high swaperror group from the corresponding value from the low swap-error group. A one-tailed was conducted with $p$ referring to the proportion of the 2500 subtractions with a value $\leq 0$. Finally, we planned to assess the test-retest reliability of $p \mathrm{~N}$ as estimated from the behavioral screening session versus $p N$ as estimated from the fMRI session, by Pearson correlation.

Hypothesis 1 of experiment 2 formalized the intuition that one would expect that individual differences in $p \mathrm{~N}$, a behavioral measure, would be reflected in neural evidence for inappropriate activation of the identity of non-probed items. To assess this prediction, as we did in experiment 1 , we operationalized "orientation recall specificity" as the difference (on 30 trials) between probe-epoch CTFs of the orientation of the probed versus of a non-probed item, then compared this measure between swap-error groups. To compute orientation recall specificity, for each trial one of the two non-probed sample items was selected at random and the amplitude of the reconstruction of the orientation of that non-probed item was subtracted from the amplitude of the reconstruction of the orientation of the probed item. Significance of all comparisons was assessed with bootstrapping. Using the results from experiment 1 as a starting point, and taking into account that fact that the modified design of experiment 2 increased effect sizes for reconstruction of stimuli from 30 trials, we determined that we required an $N$ of 10 subjects to achieve $90 \%$ power to detect an orientation recall-specificity effect with $\alpha=0.025$ (two-tailed $t$ test).

Hypothesis 2 of experiment 2 was analogous to hypothesis 1 , with the difference being that it operationalized the specificity of recall of the location of the probed item. Thus, we operationalized "location recall specificity" as the difference between probe-epoch CTFs of the location of the probed versus of a non-probed item, then compared this measure between swap-error groups. To compute location recall specificity, for each trial one of the two non-probed sample items was selected at random and the amplitude of the reconstruction of the location of this non-probed item was subtracted from the amplitude of the reconstruction of the location of the probed item. Significance of all comparisons were assessed with bootstrapping. Because experiment 1 did not use IEM to assess the neural representations of stimulus location, we used a different dataset from our lab to estimate power, and these data suggested that data from only three subjects were needed to achieve $90 \%$ power to detect a reliable location recall-specificity effect with $\alpha=0.025$ (two-tailed $t$ test).

Results from experiment 1 suggested that delay-period representation of sample locations in parietal cortex was stronger in the low swap-error group. Specifically, MVPA decoding of stimulus location was superior for the low swap-error group at TR6 and TR7. Follow-up correlational analyses indicated that individual differences in MVPA of sample location information in parietal delay-period activity correlated with $p \mathrm{~N}$ and with the location recall specificity effect. Hypothesis 3 was therefore intended to assess the prediction that individual differences in the strength of delay-period representation of the sample location in parietal cortex, as operationalized by the amplitude of the CTF of the location of the to-be-probed item at TR6 and TR7, would predict individual differences in $p N$. We planned to carry this out with Spearman correlation of rank ordering of these two variables, because subjects in the low swap-error group would necessarily have $p \mathrm{~N}$ values close to 0 . The coefficient of determination $\left(R^{2}\right)$ for this effect in experiment 1 , with an $N$ of 16 , was 0.282 . Because hypothesis 4 would also use the same measure of delay-period representation of the sample location in parietal cortex, we set $\alpha=0.025$ and calculated that, to achieve $90 \%$ power to find this effect, we would need to enroll an $N$ of 29.

Hypothesis 4 assessed the prediction that individual differences in the strength of delay-period representation of sample locations in parietal cortex, as operationalized by the amplitude of the CTF of the location of the to-beprobed item, would predict individual differences in location recall specificity. We planned to carry this out with a Pearson correlation, because we expected, based on experiment 1 , that both variables would be normally distributed. (However, if either distribution turned out to be skewed (as estimated via Shapiro-Wilk analysis), we would instead use Spearman correlation). Based on the fact that the $R^{2}$ for this effect in experiment 1 , with an $N$ of 16 , was 0.303 , we estimated that we would need $N=25$ to achieve $90 \%$ power to detect this effect at $\alpha=0.025$.

\section{Results}

\section{Experiment 1}

The only results from experiment 1 that are reported are those that were not used to generate the preregistered hypotheses, which were tested in experiment 2.

\section{Behavioral session}

Here, we report results from only the 16 subjects who also participated in the fMRI experiment. An average of 2.286 trials $(S D=2.920) ; 4.857$ trials $(S D=2.824)$, and 1.857 trials $(S D=2.824)$ per subject were excluded in the 10, 30, and 101C1L condition, respectively. Descriptive statistics suggested that task difficulty increased from 10 to $101 \mathrm{C} 1 \mathrm{~L}$ to 30 , as reflected in the mean response error $\left(F_{(2,30)}=41.830, p<0.0001\right.$; paired $t$ test, $t s>3.960$, ps $<0.002$; Table 1). Results from mixture modeling mirrored this pattern, with $\mathrm{pT}$ highest for 10 , followed by 101C1L and $30\left(F_{(2,30)}=16.791, p<0.0001\right)$, paired $t$ test $t s>2.644$, ps $<0.018$; Table 1 ). Recall precision $(\kappa)$ also differed across trials types $\left(F_{(2,30)}=16.458, p<\right.$ $0.001)$, being significantly different between 10 and 30 $\left(t_{(15)}=5.253, p<0.0001\right)$ and between 30 and $101 \mathrm{C} 1 \mathrm{~L}$ $\left(t_{(15)}=4.325, p<0.001\right)$ trials, although not differing between 10 and $101 \mathrm{C} 1 \mathrm{~L}$ trials $\left(t_{(15)}=1.387, p=0.186\right)$. Finally, although the group mean $p \mathrm{~N}$ (swap errors) on 30 trials was $0.120(S D=0.116)$, six subjects had a $p N$ at or near 0 (all $p N s<0.006$ ), indicating that these subjects made effectively no swap errors, whereas the remaining 
Table 1: Behavioral results from experiment 1 ( $n=16$ for both sessions)

\begin{tabular}{|c|c|c|c|c|c|c|}
\hline \multirow[b]{2}{*}{ Trial type } & \multicolumn{2}{|c|}{ Descriptive data } & \multicolumn{4}{|c|}{ Three-factor mixture model (parameter estimates) } \\
\hline & $\mathrm{RT}(\mathrm{s})$ & Response error (degree) & pT & $p N$ & $p U$ & $\kappa\left(\operatorname{rad}^{-1}\right)$ \\
\hline 10 & $2.825(0.247)$ & $7.395(2.360)$ & $0.987(0.019)$ & N/A & $0.013(0.019)$ & $12.899(7.706)$ \\
\hline 101C1L & $2.687(0.336)$ & $10.764(5.076)$ & $0.895(0.114)$ & N/A & $0.105(0.114)$ & $10.718(7.004)$ \\
\hline \multicolumn{7}{|c|}{ fMRI session } \\
\hline 10 & $2.912(0.149)$ & 7.765 (4.219) & $0.946(0.001)$ & $\mathrm{N} / \mathrm{A}$ & $0.054(0.001)$ & $13.748(6.881)$ \\
\hline
\end{tabular}

10 subjects all had a $p \mathrm{~N}$ of 0.127 or higher, corresponding to an average of nearly $20 \%$ swap errors on 30 trials for these ten subjects.

\section{$f M R I$ sessions}

Behavior. An average of $4.62(S D=2.07) 10$ trials was excluded, no trials for any subject for either of the other two trial types were excluded. Performance on 10 trials was highly correlated with pre-scan testing across the 16 subjects who participated in both ( $r s>0.841, p s<0.001$; Table 1).

$f M R I$. For the occipital ROI, trial-averaged signal on all three trial types showed the expected sample-related increase, return to baseline by the end of the delay period, and probe/recall-related increase. BOLD signal intensity did not differ between 30 and $101 \mathrm{C} 1 \mathrm{~L}$ trials during TRs corresponding to the encoding and delay epochs (from TR2-TR8, ts $<1.604$, ps $>0.130$ ), but was higher on 30 than $101 \mathrm{C} 1 \mathrm{~L}$ trials during the peak response to probe/ recall (TR9-TR11, ts $>2.524$, $p s<0.048$ ). BOLD signal from all three trial types did not differ from each other or from baseline at TR7 corresponding to late delay (ts $<0.806$, ps $>0.433$; Fig. $2 A)$. The ANCOVA relating delay-period BOLD signal intensity to behavioral precision revealed no significant within-subject correlations between either 10 and 30 trials or 10 and $101 \mathrm{C} 1 \mathrm{~L}$ trials (Fig. 2B).

For the parietal and frontal ROls, in both regions, trialaveraged signal on all three trial types remained elevated across the duration of the trial, with sample-related and delay-related activity greater for 30 trials than for the other two trial types (from TR3 to TR8, ts $>3.041$, $p s<0.019)$, and sample-related activity for 101C1L trials greater than for 10 trials during the encoding epoch (TR3-TR4, ts $>2.514$, ps $<0.048$ ). Beginning with TR6 in the delay period, however, BOLD signal no longer differed between 101C1L and 10 trials ( $t s<1.848$, $p s>0.138)$. That is, delay-period activity in parietal and frontal cortex was not sensitive to memory load (one item vs three items) per se, but was sensitive to stimulus category homogeneity (operationalized as $101 \mathrm{C} 1 \mathrm{~L}$ vs 30; Fig. 2A). ANCOVAs relating delay-period BOLD signal intensity to behavioral precision revealed significant within-subject correlations between 10 and 30 trials in both regions ( $r s>0.539$; $p s<0.05$ ), and a nonsignificant trend in this direction between $101 \mathrm{C} 1 \mathrm{~L}$ and 30 trials in parietal cortex $(r=0.458 ; p=0.06$. Fig. $2 B)$.

\section{Experiment 2}

\section{Behavioral}

Behavioral session. A total of 39 of 72 subjects who met a behavioral screening criterion of performing with a "low" swap error rate $(<0.05)$ or "high" swap error rate $(>0.12)$ were invited to participate two subsequent $f M R I$ sessions. From these, 29 subjects completed the two fMRI scanning sessions [low swap-error group, mean(SD): $p \mathrm{~T}=0.923(0.085), p \mathrm{~N}=0.015(0.015), p \mathrm{U}=0.062(0.080)$, $\kappa=3.844(2.402)$; high swap-error group, $p \mathrm{~T}=0.674$ (0.133), $p \mathrm{~N}=0.254(0.085), p U=0.071(0.075), \kappa=3.294$ (1.815)].

$f M R I$ session. Two subjects were excluded from the $\mathrm{fMRI}$ analyses - one because responses were not registered on $>40 \%$ trials, and one because mixture modeling indicated that $p U$ was $>0.75$ (outside three SD from the mean value), and so the data from 27 are reported here. The behavioral performance of many of these 27 subjects, specifically their $p \mathrm{~N}$, differed considerably during the scanning sessions in comparison to the behavioral screening session, with eight subjects initially classified as "low swap error" having $p \mathrm{~N}$ values $>0.05$ and one subject initially classified as "high swap error" having a $p \mathrm{~N}$ value $<0.12$. A consequence of this that the same sample of subjects who had been selected to form a bimodal distribution of "extreme" values of $p \mathrm{~N}$ yielded a continuous distribution of values from the $\mathrm{fMRI}$ session, with 15 subjects with $p \mathrm{~N}$ values $<0.05$, six with $p \mathrm{~N}$ values $>0.05$ and $<0.12$, and eight with $p \mathrm{~N}$ values $>0.12$ (Fig. 2). As a consequence, with too few high swap-error subjects, we were unable to test our hypotheses with the preregistered analyses. We reasoned, however, that the original intent of the preregistered hypothesis tests would be met if we deviated from the preregistered criteria for classifying subjects and, instead, conducted the planned analyses on two groups determined by a median split of $p \mathrm{~N}$ values. From the perspective of scientific publishing, this deviation from the preregistered plan would qualify these analyses as "exploratory" were this a formal Registered Report. From the perspective of our experimental design, it might be expected a priori to decrease the power of the design, because several subjects in the high swap-error group had with $p \mathrm{~N}$ values lower than the lower bound of $p \mathrm{~N}>0.12$. that had been used to calculate power.

\section{Analyses of data from $\mathrm{fMRI}$ session grouped by median split}

The median split assigned 14 subjects, with $p N s$ ranging from 0 to 0.039 , to the low swap-error group (mean 


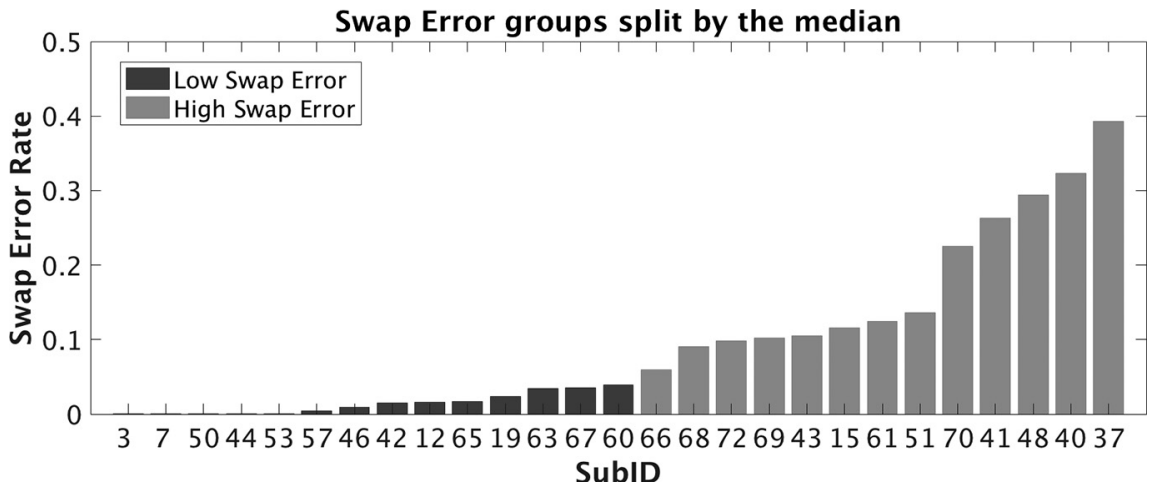

Figure 3. Swap error rates for each subject in the fMRI session of experiment 2. Low swap-error subjects (dark gray) and high swap-error subjects (light gray) were defined via median split.

(SD): $p \mathrm{~T}=0.896(0.127), p \mathrm{~N}=0.012(0.013), p \mathrm{U}=0.091(0.125)$, and $\kappa=3.092(1.498)$, and 13 subjects, with $p N s$ ranging from 0.059 to 0.393 , to the high swap-error group [mean(SD): $p \mathrm{~T}=0.700(0.170), \mathrm{pN}=0.169(0.109), p \mathrm{U}=0.131(0.177)$, and $\kappa=3.652(1.881$ ); Fig. 3). Independent $t$ tests between the groups revealed no significant difference in $p U$ or $\kappa$ (ts $<0.851, p s>0.403)$. Test-retest stability between the behavioral-screening and fMRI sessions was assessed with correlation analyses. Pearson correlations revealed significant test-retest correlations for $p T$ and for $\kappa\left(R^{2}>0.477, p s<0.001\right)$. Because $p \mathrm{~N}$ from the behavioral screening session was selected to be dichotomous, a Spearman correlation was conducted on this measure, and it failed to provide evidence for a correlation of performance across the two sessions $\left(R^{2}=\right.$ $0.054, p=0.247$ ).

\section{Hypothesis tests}

Hypothesis 1. In the occipital ROI, at TR10, for the low swap-error group, IEM reconstruction of stimulus orientation on 30 trials was significantly larger than baseline $(p=0.047$; Fig. $4 A)$, thereby meeting the prerequisite for being able to test hypothesis 1 . (Neither the comparable result for the high swap-error group, nor the reconstructions of the orientation of a non-probed item for either group, approached significance; ps $>0.671$; Fig. $4 A$ ). Comparison of orientation recall specificity between the two groups indicated that this measure was higher for the low swap-error group than for the high swap-error $(p=0.049$; Fig. 4B), and thus supported hypothesis 1 .

Hypothesis 2. In the occipital ROI, at TR10, IEM reconstruction of the probed location on 30 trials was significantly larger than baseline for both the low swap-error group $(p<0.001)$ and the high swap-error group $(p=0.021$; Fig. 5A). Comparable reconstructions for non-probed locations were nonsignificant for both groups (low swap error, $p=0.059$ (trending toward negative); high swap error $p=0.770$; Fig. $5 A$ ). Comparison of location recall specificity between the two groups indicated that although this measure was numerically higher for the low swap-error group than for the high swap-error group (Fig. 5B), this difference approached, but did not achieve, the threshold for significance $(p=0.067)$.

Hypothesis 3. IEM did not yield reliable reconstruction of the to-be-probed location in the parietal ROI during TR6 and TR7 of the delay period for either group (ps $>0.213$ ), and so a direct test of hypothesis 3 , as it was preregistered, could not be conducted. (Note that the exclusion of two subjects was not factor here, because the planned $N$ of 29 was needed for the planned correlation,
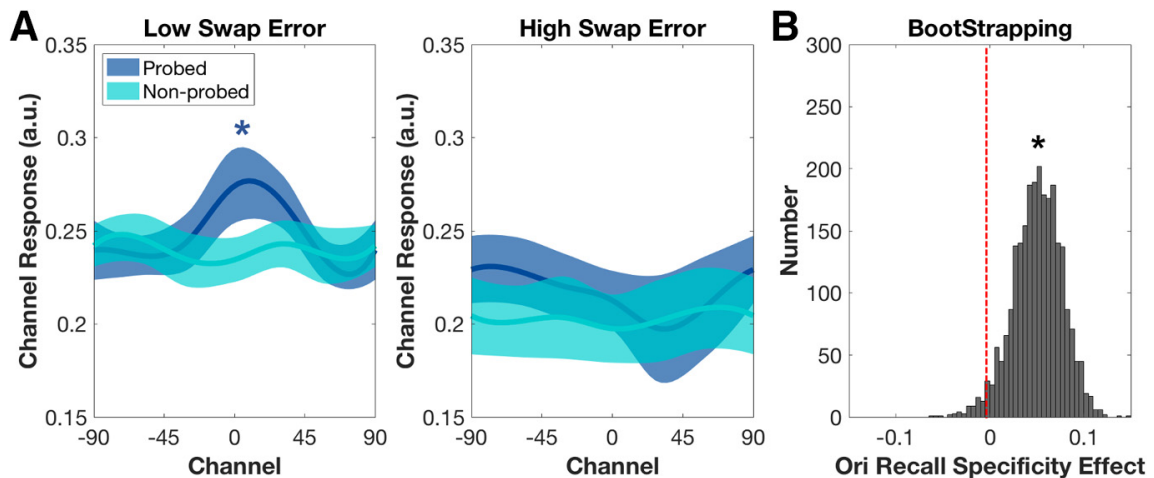

Figure 4. Orientation recall specificity effect in the occipital ROI, at TR10 on 30 trials (experiment 2). $\boldsymbol{A}$, Neural reconstructions for probed and non-probed orientations in the two groups. Asterisks indicate significant reconstructions at $p<0.05$. $\boldsymbol{B}$, Distributions of bootstrapping estimates of orientation recall-specificity effects. The recall specificity effects were identified by the recall specificity difference between low and high swap error group. Asterisk indicates significant difference between the two distributions $(p<0.05)$. 

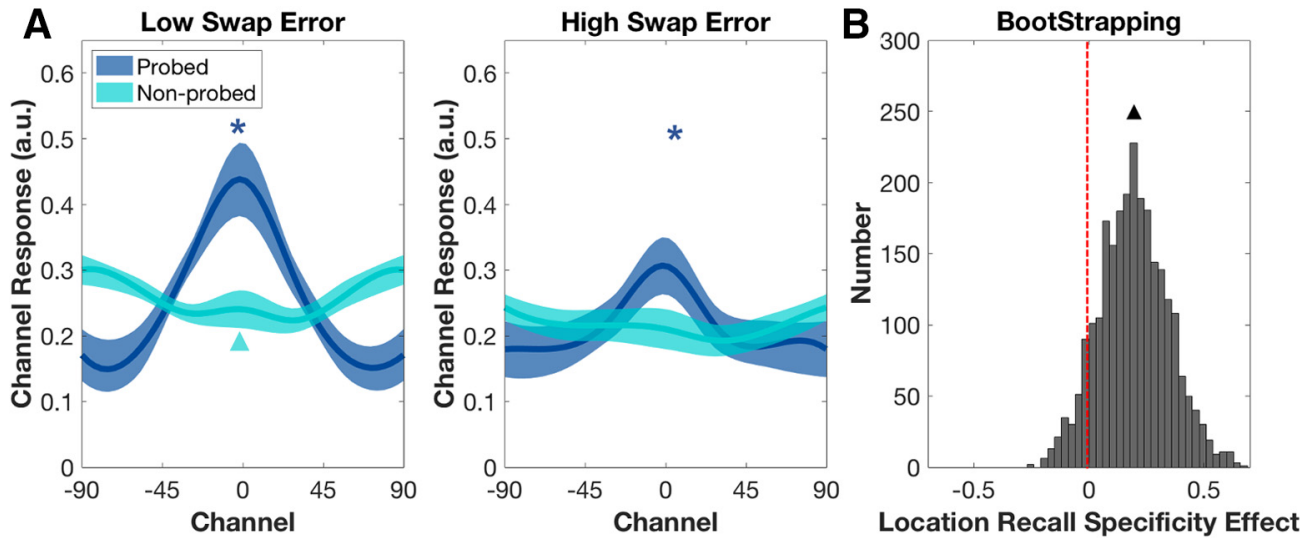

Figure 5. Location recall specificity effect in the occipital ROI, at TR10 on 30 trials (experiment 2). $\boldsymbol{A}$, Neural reconstructions for probed and non-probed locations in the two groups. Asterisks indicate significant reconstructions $(p<0.05)$, and triangle indicates trend-level evidence for a significantly negative-going reconstruction $(0.05<p<0.1)$. $\boldsymbol{B}$, Distributions of bootstrapping estimates of location recall-specificity effects in the two groups. Triangle indicates trend-level evidence for a difference between the two distributions $(0.05<p<0.1)$.

not for the reliable reconstruction of the to-be-probed location, which would have been expected to require considerably fewer subjects). When we nevertheless conducted the planned Spearman correlation with data from TR6 and TR7, it was not significant $(p>0.23)$. However, because the reconstruction of stimulus location was significant for the low swap-error group at adjacent TR8 $(p=0.041)$, we applied the procedures for testing hypothesis 3 to this adjacent time point, as an exploratory analysis. Location representation-specificity was reliable at TR8 for the low swap-error group $(p=0.041)$ but not for the high swaperror group $(p=0.566)$. Across all subjects, Spearman correlation indicated that higher values of location representation-specificity in IPS were associated with lower values of $p \mathrm{~N}\left(R^{2}=0.269, p=0.01\right)$.

Hypothesis 4. For the same reason as hypothesis 3, a direct test of hypothesis 4 , as it was preregistered, could not be conducted. Thus, analogous to hypothesis 3 , we also applied the procedures for testing hypothesis 4 to TR8, as an exploratory analysis. Across all subjects, Spearman correlation indicated that higher values of location representation-specificity in IPS were associated with higher values of location recall specificity in occipital cortex, at $\operatorname{TR} 10\left(R^{2}=0.357, p=0.001\right)$.

\section{Secondary hypotheses}

$B O L D$ signal intensity. In all three ROls, for 10 and 30 trials, the time course of $\mathrm{fMRI}$ activity was comparable to what was observed in experiment 1: In the occipital ROI, at TR7, signal in the occipital ROI did not differ between 30 and 10 trials $\left(t_{(26)}=1.617, p=0.118\right)$; whereas it was significantly higher for 30 than for 10 trials in the parietal and frontal ROls (ts $>3.572$, ps $<0.001$ ). Furthermore, the ANCOVAs relating load-related changes in signal at TR7 to load-related changes in behavioral recall precision was not significant in the occipital ROI, but the analogous ANCOVAS were significant in both the parietal $\left(R^{2}=0.48\right)$ and frontal $\left(R^{2}=0.4 ; p s<0.001\right)$ ROls.

The time course of IEM reconstructions of orientation during 30 trials. In the occipital $\mathrm{ROI}$, the reconstruction of the to-be-probed orientation was successful during the sample-presentation and recall epochs (low swap-error group: TR4 and TR10; high swap-error group: TR4 and TR8; $p$ values for these IEM reconstructions not FDR-corrected), but not during the delay period. Orientation could not be reconstructed in the parietal and frontal ROls (Fig. $6 A$ ). (Note that it has previously been reported that, on 10 trials, when collapsing across all subjects, orientation can be reconstructed during the entirety of the trial in each of these three regions; Cai et al., 2019.)

Turning to the reconstruction of the location of the probed item, in the occipital ROI location could be reconstructed across the entirety of the trial in both groups, with reconstruction amplitude significantly greater for the low swap-error group during portions of sample presentation (TR3) and recall (TR10 and TR11) epochs, and trending in this direction during the delay period (TR6 and TR7; Fig. $6 B$ ). In the parietal $\mathrm{ROI}$, location could be reconstructed during the sample presentation (TR3 and TR4) and during recall (TR8-TR11) in both groups, with a trend toward greater amplitude in the low swap-error group at TR7 and TR8 (Fig. 6B). In the frontal ROI location reconstruction was only successful at one time point during recall (TR8) in the low swap-error group (Fig. 6B).

\section{Discussion}

Success on VWM tasks that are nominally nonspatial can often nonetheless require memory for the location at which each item was presented, and thus require context binding (compare Olson and Marshuetz, 2005; Jiang et al., 2009). The results presented here are consistent with the proposition that delay-period activity of the IPS may correspond more closely to the operation of context binding than to the representation of stimulus identity per se. Experiment 1 varied the demands on context binding by using two types of three-item trials, 30 trials that could only be solved if the subject remembered which item had been presented at the location of the recall dial, and 101C1L trials in which memory for item-location 
A

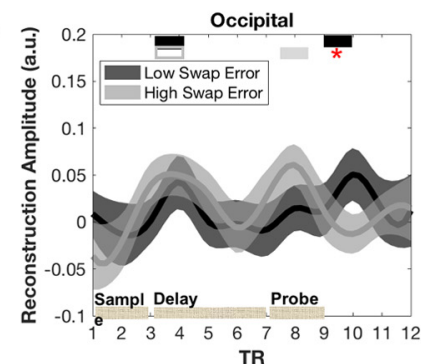

B

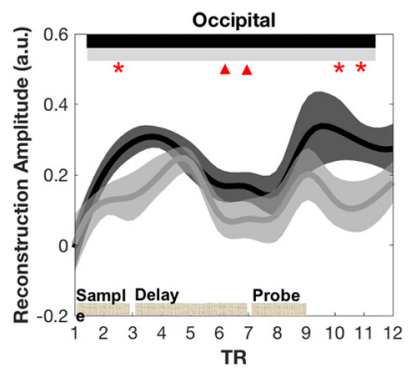

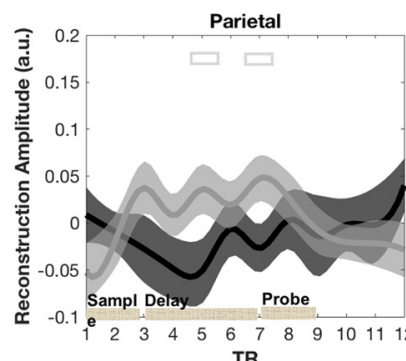

TR

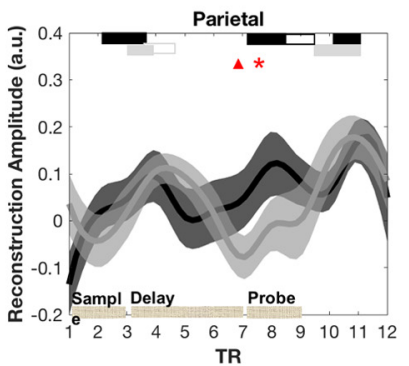

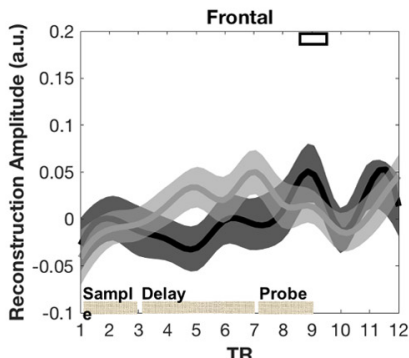

TR

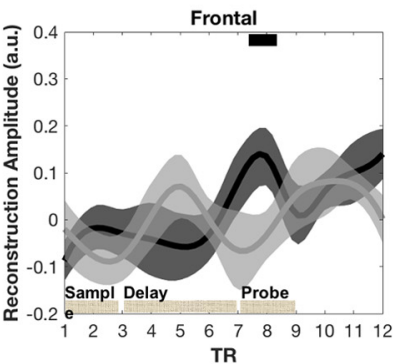

Figure 6. Time course of IEM reconstructions of unshifted data from 30 trials. $\boldsymbol{A}$, Reconstructions of the orientation of the probed stimulus broken out by swap-error group, in the three ROls. $\boldsymbol{B}$, Reconstruction of the location of the probed stimulus broken out by swap-error group, in the three ROls. Bars along the top indicate values statistically different from baseline, with color corresponding to swap-error group; * indicates statistical difference between swap-error group at that TR (not FDR-corrected). Bars along the bottom indicate the timing of trial epochs.

associations was not needed, because the nonspatial features of the recall dial uniquely indicated which item was to be recalled. The delay-period BOLD response in IPS (and in frontal cortex) was sensitive to demands on context binding (30 vs $101 \mathrm{C} 1 \mathrm{~L})$, but not to the number of items that needed to be remembered (10 vs 101C1L; Fig. 2). This finding replicates and extends a previous finding (Gosseries et al., 2018), and has at least two implications for our understanding of VWM. First, it suggests that the interpretation of results from many previous reports of load-sensitive activity in IPS (Todd and Marois, 2004, 2005; Xu and Chun, 2006) may need to be revisited, because of the possibility that such studies have often confounded the factors of load and of context binding. Second, it provides an explicit, mechanistic account of the "benefit of memory set heterogeneity" (Olson and Jiang, 2002; Delvenne and Bruyer, 2004), and may provide support for models that explicitly incorporate context binding (Swan and Wyble, 2014; Oberauer and Lin, 2017; Bouchacourt and Buschman, 2019) as well as a challenge for models that do not (including slot and resource models; compare Oberauer et al., 2016).

The results from experiment 2 broadly replicated the intriguing preliminary findings from experiment 1 that individual differences in the rate of swap errors were associated with neural measures of the strength of the representation of the (nonspatial) orientation and the location of stimuli. Low swap-error subjects showed higher orientation recall specificity in occipital cortex (hypothesis 1 ), and a trend in the same direction for location recall specificity (hypothesis 2). These results provide a demonstration, at the neural level, for a phenomenon predicted by a theoretical and computational model of VWM: stronger context binding should result in a lower level of competition between the probed item and nonprobed items at the time of retrieval (Oberauer and Lin, 2017).

For IPS, exploratory results from experiment 2 showed a pattern comparable to what was seen experiment 1, but at a later time point: the strength of delay-period representation of stimulus location (i.e., of context) in IPS predicted lower swap-error rates (hypothesis 3), and higher location recall specificity in occipital cortex (hypothesis 4). These are consistent with the proposal from Gosseries et al. (2018) that delay-period activity in IPS may be particularly important for the maintenance of the bindings of context to content. We note that whereas the present results are consistent with the proposed functions of a spatial priority map (Bisley and Goldberg, 2010; Jerde et al., 2012; Bisley and Mirpour, 2019), the task-critical context in Gosseries et al. (2018) was ordinal position. Thus, an important question for future research is determining whether the processing of context in IPS is conducted in a domain-specific or a domain-general manner.

Interpretation of some of the results from experiment 2 is complicated by a pattern in the results of this multisession experiment that was unanticipated: subject's swaperror rates $(p N)$ were less stable between the behavioral screening session and the $\mathrm{fMRI}$ sessions than were other indices of performance, including precision $(\kappa)$ and the probability of a target response (DT). As a result, several subjects selected for the low swap-error group based on performance at behavioral screening performed the task with a higher rate of swap errors during the $\mathrm{fMRI}$ sessions. The resultant decrease in sensitivity relative to what had been expected for the preregistered extreme-groups design might explain why the between-group difference in location recall specificity did not achieve statistical significance. There are at least two possible explanations for 
the greater volatility of estimates of $p \mathrm{~N}$ than those of $\kappa$ and of $p T$. The less interesting possibility is a technical one: model estimates of $p \mathrm{~N}$ may simply be less robust than those of other parameters, particularly at the relatively small number of trials that features in the fMRI sessions from experiment 2. (Note, however, that we did try two other models that we only became aware of after preregistering experiment 2 (Bays, 2016; Oberauer et al., 2017), but these did not produce appreciably different results). The potentially more interesting possibility is that the hypothesized mechanism that is indexed by $p \mathrm{~N}$, context binding, may be more sensitive to challenging testing conditions (e.g., the MRI scanner session vs the behavioral lab) than are the mechanisms underlying $\kappa$ and $p T$.

\section{References}

Bays P (2016) Evaluating and excluding swap errors in analogue tests of working memory. Sci Rep 6:19203.

Bays PM, Catalao RFG, Husain M (2009) The precision of visual working memory is set by allocation of a shared resource. $\mathrm{J}$ Vis 9:7.1-11.

Bettencourt KC, Xu Y (2016) Decoding the content of visual shortterm memory under distraction in occipital and parietal areas. Nat Neurosci 19:150-157.

Bisley JW, Goldberg ME (2010) Attention, intention, and priority in the parietal lobe. Annu Rev Neurosci 33:1-21.

Bisley JW, Mirpour K (2019) The neural instantiation of a priority map. Curr Opin Psychol 29:108-112.

Bland JM, Altman DG (1995) Comparing methods of measurement: why plotting difference against standard method is misleading. Lancet 346:1085-1087.

Bouchacourt F, Buschman TJ (2019) A flexible model of working memory. Neuron 103:147-160.

Brainard DH (1997) The psychophysics toolbox. Spat Vis 10:433436.

Brouwer GJ, Heeger DJ (2009) Decoding and reconstructing color from responses in human visual cortex. J Neurosci 29:1399214003.

Brouwer GJ, Heeger DJ (2011) Cross-orientation suppression in human visual cortex. J Neurophysiol 106:2108-2119.

Cai Y, Sheldon AD, Yu Q, Postle BR (2019) Overlapping and distinct contributions of stimulus location and of spatial context to nonspatial visual short-term memory. J Neurophysiol 121:1222-1231.

Cox RW (1996) AFNI: software for analysis and visualization of functional magnetic resonance neuroimages. Comput Biomed Res 29:162-173.

Delvenne JB, Bruyer R (2004) Does visual short-term memory store bound features? Vis Cogn 11:1-27.

Emrich SM, Riggall AC, Larocque JJ, Postle BR (2013) Distributed patterns of activity in sensory cortex reflect the precision of multiple items maintained in visual short-term memory. J Neurosci 33:6516-6523.

Ester EF, Sprague TC, Serences JT (2015) Parietal and frontal cortex encode stimulus-specific mnemonic representations during visual working memory. Neuron 87:893-905.

Ester EF, Sutterer DW, Serences JT, Awh E (2016) Feature-selective attentional modulations in human frontoparietal cortex. J Neurosci 36:8188-8199.
Fukuda K, Woodman G, Vogel E (2015) Individual differences in visual working memory capacity: contributions of attentional control to storage. In: Mechanisms of sensory working memory: attention and performance (Jolicouer P, Lefebvre B, Martinez-Trujillo J, eds). San Diego: Academic Press.

Gosseries O, Yu Q, LaRocque JJ, Starrett MJ, Rose N, Cowan N, Postle BR (2018) Parietal-occipital interactions underlying controland representation-related processes in working memory for nonspatial visual features. J Neurosci 38:4357-4366.

Jerde T, Merriam EP, Riggall AC, Hedges JH, Curtis CE (2012) Prioritized maps of space in human frontoparietal cortex. J Neurosci 32:17382-17390.

Jiang YV, Makovski T, Shim WM (2009) Visual memory for features, conjunctions, objects, and locations. In: The visual world in memory. (Brockmole JR, ed), pp 33-65. East Sussex: Psychology Press.

Oberauer K, Lin HY (2017) An interference model of visual working memory. Psychol Rev 124:21-59.

Oberauer K, Farrell S, Jarrold C, Lewandowsky S (2016) What limits working memory capacity? Psychol Bull 142:758-799.

Oberauer K, Stoneking C, Wabersich D, Lin HY (2017) Hierarchical Bayesian measurement models for continuous reproduction of visual features from working memory. J Vis 17:11.

Olson IR, Jiang Y (2002) Is visual short-term memory object based? Rejection of the "strong-object" hypothesis. Percept Psychophys 64:1055-1067.

Olson IR, Marshuetz C (2005) Remembering "what" brings along "where" in visual working memory. Percept Psychophys 67:185194.

Schneegans S, Bays PM (2017) Neural architecture for feature binding in visual working memory. J Neurosci 37:3913-3925.

Serences JT, Saproo S (2012) Computational advances towards linking BOLD and behavior. Neuropsychologia 50:435-446.

Sprague TC, Adam KCS, Foster JJ, Rahmati M, Sutterer DW, Vo VA (2018) Inverted encoding models assay population-level stimulus representations, not single-unit neural tuning. eNeuro 5:ENEURO. 0098-18.2018.

Swan G, Wyble B (2014) The binding pool: a model of shared resources for distinct items in visual working memory. Atten Percept Psychophys 76:2136-2157.

Todd JJ, Marois R (2004) Capacity limit of visual short-term memory in human posterior parietal cortex. Nature 428:751-754.

Todd JJ, Marois R (2005) Posterior parietal cortex activity predicts individual differences in visual short-term memory capacity. Cogn Affect Behav Neurosci 5:144-155.

Unsworth N, Fukuda K, Awh E, Vogel EK (2014) Working memory and fluid intelligence: capacity, attention control, and secondary memory retrieval. Cogn Psychol 71:1-26.

Vogel EK, McCollough AW, Machizawa MG (2005) Neural measures reveal individual differences in controlling access to working memory. Nature 438:500-503.

$\mathrm{Xu}$ Y (2017) Reevaluating the sensory account of visual working memory storage. Trends Cogn Sci 21:794-815.

Xu Y, Chun MM (2006) Dissociable neural mechanisms supporting visual short-term memory for objects. Nature 440:91-95.

Yarkoni T, Braver TS (2010) Cognitive neuroscience approaches to individual differences in working memory and executive control: Conceptual and methodological issues. Handbook of individual differences in cognition: attention, memory, and executive control, pp 87-107. New York: Springer Science + Business Media.

Yu Q, Shim WM (2017) Occipital, parietal, and frontal cortices selectively maintain task-relevant features of multi-feature objects in visual working memory. Neuroimage 157:97-107. 